\title{
Effect of Oblique X-ray Incidence in Flat-Panel Computed Tomography of the Breast
}

\author{
Aldo Badano*, Iacovos S. Kyprianou, Melanie Freed, Robert J. Jennings, and Josep Sempau
}

\begin{abstract}
We quantify the variation in resolution due to anisotropy caused by oblique $\mathrm{X}$-ray incidence in indirect flat-panel detectors for computed tomography breast imaging systems. We consider a geometry and detector type utilized in breast computed tomography (CT) systems currently being developed. Our methods rely on MANTIS, a combined X-ray, electron, and optical Monte Carlo transport open source code. The physics models are the most accurate available in general-purpose Monte Carlo packages in the diagnostic energy range. We consider maximum-obliquity angles of $10^{\circ}$ and $13^{\circ}$ at the centers of the 30 and $40 \mathrm{~cm}$ detector edges, respectively, and $16^{\circ}$ at the corner of the detector. Our results indicate that blur is asymmetric and that the resolution properties vary significantly with the angle (or location) of incidence. Our results suggest that the asymmetry can be as high as a factor of 2.6 between orthogonal directions. Anisotropy maps predicted by MANTIS provide an understanding of the effect that such variations have on the imaging system and allow more accurate modeling and optimization of breast CT systems. These maps of anisotropy across the detector could lead to improved reconstruction and help motivate physics-based strategies for computer detection of breast lesions.
\end{abstract}

Index Terms-Breast computed tomography (CT), Cesium Iodide, Monte Carlo simulation, phosphor blur, phosphor screen.

\section{INTRODUCTION}

$\mathbf{T}$ HREE-DIMENSIONAL imaging of the breast has the potential to improve detectability of breast cancer lesions by removing the overlaying normal anatomical background that complicates the visual task in mammography. Among the 3-D technologies currently under development, breast tomosynthesis [1] and breast computed tomography [2], [3] have already shown promising results [4]-[6]. However, the design and optimization of 3-D breast imaging systems requires an

Manuscript received June 19, 2008; revised October 30, 2008. First published December 09, 2008; current version published April 29, 2009. The work of J. Sempau was supported by the Spanish Ministerio de Educación y Ciencia under Project FIS 2006-07016. The mention of commercial products herein is not to be construed as either an actual or implied endorsement of such products by the Department of Health and Human Services. Asterisk indicates corresponding author.

*A. Badano is with the NIBIB/CDRH Laboratory for the Assessment of Medical Imaging Systems, Division of Imaging and Applied Mathematics, Office of Science and Engineering Laboratories, Center for Devices and Radiological Health, U.S. Food and Drug Administration, Silver Spring, MD 20993 USA (e-mail: aldo.badano@fda.hhs.gov).

I. S. Kyprianou, M. Freed, and R. J. Jennings are with the NIBIB/CDRH Laboratory for the Assessment of Medical Imaging Systems, Division of Imaging and Applied Mathematics, Office of Science and Engineering Laboratories, Center for Devices and Radiological Health, U.S. Food and Drug Administration, Silver Spring, MD 20993 USA.

J. Sempau is with the Spanish Networking Research Center on Bioengineering, Biomaterials and Nanomedicine (CIBER-BBN), 20018 Zaragoza, Spain.

Digital Object Identifier 10.1109/TMI.2008.2010443 in-depth understanding through theory, simulation, and experimentation of the underlying physical processes that determine image quality. This can be achieved with realistic models for the different system components that incorporate all sources of image quality degradation.

In a previous paper [7], we described the effect of oblique $\mathrm{X}$-ray incidence in breast tomosynthesis systems. Our results are consistent with experimental measurements reported by Mainprize et al. [8] which show significant decreases in modulation transfer functions under oblique X-ray incidence angle for an indirect full-field digital mammography detector. In his study, Mainprize concludes that this reduction in resolution will introduce misregistration and additional noise in breast tomosynthesis reconstructions. A similar effect may be present in breast computer tomography (CT). It is the goal of this paper to report simulation results that describe the anisotropy in the response of indirect detectors used for breast CT systems at energies that are relevant for current designs.

The imaging performance of breast CT systems has been studied by many authors [4], [6], [9]. However, to date, a detailed study on the variations in imaging performance in the projection space due to oblique $\mathrm{X}$-ray incidence including spatial blur for breast CT has not been published. In breast CT, primary or unscattered X-rays hit the detector at an angle that depends on the relative position of the emission at the X-ray tube and the landing location in the detector plane. X-rays arrive at the detector after traversing various materials (including the breast) at the same oblique angle. In a cone-beam breast CT system, the angles at which X-ray beams enter the digital detector are a function of the detector size and of the distance between the source of X-rays and the imaging acquisition device. In cone-beam computed tomography applications, detectors can measure $30-43 \mathrm{~cm}$ with a highly variable source-to-detector distance between 70 and $150 \mathrm{~cm}$. These last systems utilize phosphor thicknesses typically between 400 and $600 \mu \mathrm{m}$.

For the purpose of this analysis, we used a geometric design following Kwan et al. [6] with a detector size of $30 \times 40 \mathrm{~cm}$, a source-to-isocenter distance of $44 \mathrm{~cm}$, and a source-to-detector distance of $88 \mathrm{~cm}$. This geometry results in maximum-obliquity angles of $10^{\circ}$ and $13^{\circ}$ at the centers of the 30 and $40 \mathrm{~cm}$ detector edges, respectively, and $16^{\circ}$ at the corners of the detector [7]. In indirect digital detectors where the X-ray energy gives rise to optical radiation that is subsequently detected by a photo-diode sensor layer, the path length of individual X-rays depends on the thickness of the scintillator layer. Currently, digital detectors for breast $\mathrm{CT}$ have a $\mathrm{CsI}(\mathrm{Tl})$ phosphor thickness of several hundred microns. For the purpose of this analysis, we used a phosphor thickness of $600 \mu \mathrm{m}$.

In this paper, we simulate a breast CT geometry and demonstrate that imaging performance of the indirect detectors based 
on columnar $\mathrm{CsI}(\mathrm{Tl})$ phosphors is shift variant due to oblique $\mathrm{X}$-ray incidence and the associated transport of quanta (both $\mathrm{X}$-rays and optical photons) through the phosphor layer.

\section{METHODS}

\section{A. Computational Model}

Our simulations are performed using the code MANTIS (Monte Carlo X-ray electroN opTical Imaging Simulation) that carries out the transport of X-ray photons, electrons, and optical photons within the same geometry. The code MANTIS is an extension of PENELOPE [10], [11] (an X-ray/electron Monte Carlo transport code) for optical transport using the physics of DETECT-II (an optical photon Monte Carlo transport code). The MANTIS source code is available via anonymous $\mathrm{ftp}^{1}$ It is intended for the study of problems involving indirect imaging sensors in which light is generated by the interaction of particles with matter in a transducer (so called phosphor or scintillator) material. In MANTIS, the processes of energy transfer from secondary electrons to light are modeled event by event. The details of the simulation code have been published elsewhere [12], [13]. A brief summary of its main features are presented below for completeness.

For the transport of ionizing radiation, the validity of MANTIS extends from energies above $50 \mathrm{eV}$ and up to $1 \mathrm{GeV}$, and to optical photons whose wavelength is small compared to the smallest characteristic dimension of the structures to be modeled. MANTIS includes all the relevant X-ray, and electron and positron interactions in the considered energy range from the PENELOPE package. Photon transport is simulated by means of the detailed simulation scheme, i.e., interaction by interaction. Electron and positron histories are generated on the basis of a mixed procedure, which combines detailed simulation of hard events (those involving energy losses or angular deflections above certain user-defined cutoffs) with condensed simulation of soft interactions. Within the PENELOPE package, the geometry routines (PENGEOM) permit the generation of random electron-photon showers in material systems consisting of homogeneous bodies bounded by quadratic surfaces. This package allows for translation and rotation of bodies, which will prove useful for the simulation of many-angle projection imaging systems like computed tomography and tomosynthesis.

The detailed description of the physics models can be found in [10] which is published and freely distributed with the PENELOPE code by the Nuclear Energy Agency (NEA). PENELOPE has been applied to many problems [14]-[21] and has been validated extensively [22]-[24].

PENELOPE was adapted to the specific requirements of the MANTIS simulation using PENEASY, a generic main program and accessory routines that allow easy configuration of PENELOPE. PENEASY is freely available. ${ }^{2}$

The optical transport models in MANTIS are taken from DETECT-II, an optical Monte Carlo simulation code for the study of light transport processes within emissive structures [25]-[29].

${ }^{1} \mathrm{ftp}: / / 150.148 .3 .24 /$ mantis

${ }^{2}$ http://www.upc.es/inte/downloads/penEasy.htm
DETECT-II extended previous work on optical Monte Carlo [30] by improving the description of optical physics models. Optical scattering processes described in MANTIS include bulk absorption, scattering, and Fresnel refraction [31]. The optical transport methods neglect the effect of finite wavelength, model light rays as single quanta, and interpret the reflection and transmission coefficients as probabilities [32].

When energy is deposited in the scintillator, a number of light photons are generated at that location with a specific model for the optical gain. For research purposes, MANTIS version 1.0 defines three different models for the distribution of the optical gain: zero-variance, Poisson, and Gaussian. In a nonabsorbent screen with an ideal sensor (i.e., a screen with an optical collection and optical detection efficiency of 100\%), the number of optical photons $q$ generated at each event depositing an energy $e$ is obtained by sampling $\mathcal{P}(e \Gamma)$, where $\Gamma$ is the optical gain (55 $\mathrm{keV}^{-1}$ for CsI:Tl) and $\mathcal{P}(x)$ is a Poisson distribution with mean value $x$.

The optical photons are then tracked until they reach an absorption event which in some cases contributes to the output signal. Individual optical photons that reach the photodiode undergo a test for the photo-detection process, by comparing a random number uniformly distributed in $(0,1)$ to the probability of detection, which corresponds to the spectral sensitivity at the energy of the optical photon $(E)$. If detected, the photon generates a charge (or signal) $s$ given by $s=\mathcal{P}(E / \epsilon)$, where $\epsilon$ is the energy required to generate a single electron-hole pair in the photodiode material (for a-Si:H, this value is $3.6 \mathrm{eV}$ ). The accumulated signal $s$ is binned in space to obtain the point-response function (PRF), or in pulse-height mode, to obtain the pulse-height spectrum (PHS).

\section{B. Phosphor Model}

The columnar model, improved with respect to our previous work [31], is based on the generation of geometries using quadratic surfaces and bodies from the PENGEOM routines, one of the elements included in the PENELOPE package. The screen is defined by cloning a central cell as many times as necessary. The columns, $9 \mu \mathrm{m}$ in diameter, are arranged in a matrix of square cells with a column in its center and four columns centered on its vertices. Our model of the columnar phosphor, shown in Fig. 1, consists of a scintillator crystal, a backing material, a photodiode layer, and a glass substrate. The material in between the columns is Nitrogen gas. Table I lists the material properties for each of these layers and the parameters for the phosphor model. The X-ray and electron transport cross sections were obtained using the material program included in the PENELOPE package. The optical properties of the backing and the glass substrate are irrelevant since reflector (or absorptive) surfaces are placed at the entrance of these slabs. Therefore, no optical photon travels into those layers. We used an a-Si:H photo-diode layer with a spectral sensitivity given in [33]. The reflection coefficients used in our columnar phosphor model are 1 for the reflectivity of the backing surface, and 0.2 for the reflectivity of sensor surface [7]. The surfaces of the column walls were not considered smooth and were allowed to 


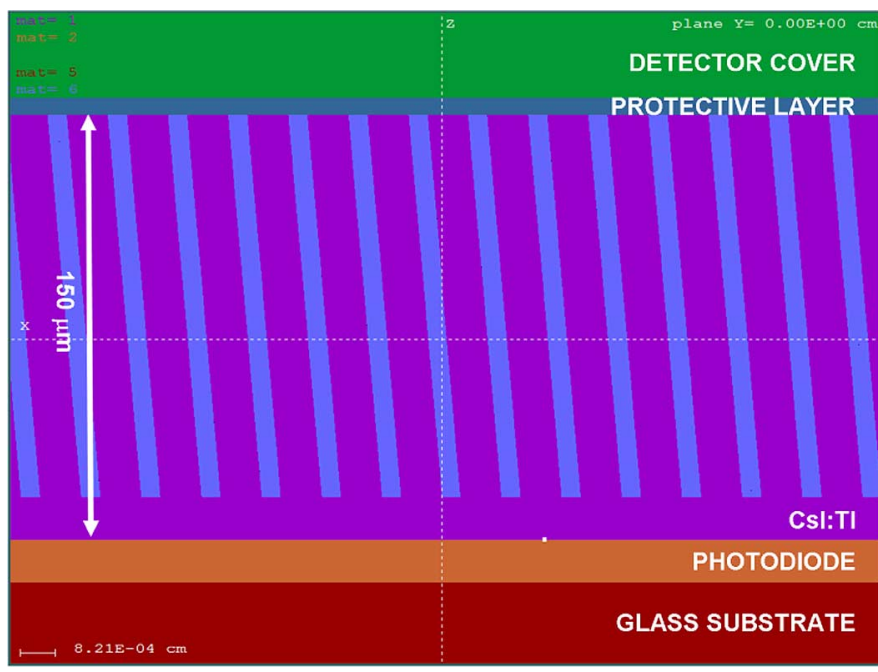

Fig. 1. Cross-section illustrating the phosphor structures included in the geometric model for the MANTIS Monte Carlo simulations. All dimensions are to scale except the top (detector cover) and bottom (glass substrate) slabs which are only showed partially in order to make the details of smaller structures (including the scintillator columns) visible in the diagram.

TABLE I

Parameters of the Columnar Csi(Tl) Phosphor Model

\begin{tabular}{|c|c|c|c|}
\hline \multicolumn{4}{|c|}{$\begin{array}{l}\text { Columnar structure: diameter } 9 \mu \mathrm{m}, \\
\text { packing density } 85 \% \text { (approx.), } 5^{\circ} \text { tilt } \\
\text { and } 15 \% \text { (of the thickness) unstructured base }\end{array}$} \\
\hline \\
\hline \multicolumn{3}{|l|}{ Scintillator } & 600 \\
\hline \multicolumn{3}{|l|}{ Photodiode } & 10 \\
\hline \multicolumn{3}{|c|}{ Protective layer (transparent acrylic) } & 1 \\
\hline \multicolumn{3}{|l|}{ Substrate (glass) } & 1000 \\
\hline \multicolumn{3}{|l|}{ Backing (a-C) } & 2000 \\
\hline \multicolumn{3}{|l|}{$\mathrm{Ni}$ gas } & setween columns \\
\hline Optical properties & $n$ & $\mu_{a b s}\left(\mathrm{~cm}^{-1}\right)$ & $\mu_{s c a}\left(\mathrm{~cm}^{-1}\right)$ \\
\hline Scintillator & 1.8 & 1.0 & 1.0 \\
\hline Gas & 1.0 & 0.0 & 0.0 \\
\hline Photodiode & 1.7 & 0.1 & 10.0 \\
\hline
\end{tabular}

have a fixed variability in the surface normal according to the algorithm described in [12].

In terms of the possible validation approaches that can be used to understand the ability of MANTIS to predict spatial spread of the signal due to particle and optical transport in columnar scintillators, we have focused the validation of detector models on matching several parameters that represent a more complete description of the phosphor being modeled [7], [34]. The three performance parameters we have previously used are the information (Swank) factor $I$, the light output (either in absolute or relative scales depending on the availability of data from the literature), and the spatial detector response. A full report on the validation of the simulation results with measurements of phosphor blur for a variety of screen designs, thicknesses, and X-ray energies will be published elsewhere [35] (preliminary results were included in [7]).

The comparison of the simulation results with measured response functions requires an experimental method that can capture asymmetry. Thus, as has been reported by Kyprianou $e t$ al . [36], the traditional methods for estimating resolution that rely on edges or slits are not useful in this case. Our approach relies

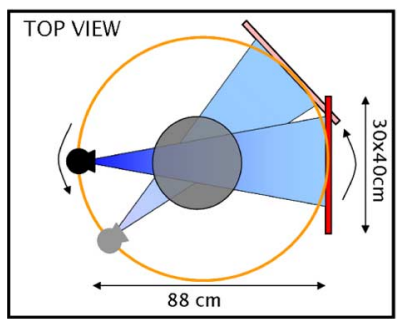

(a)

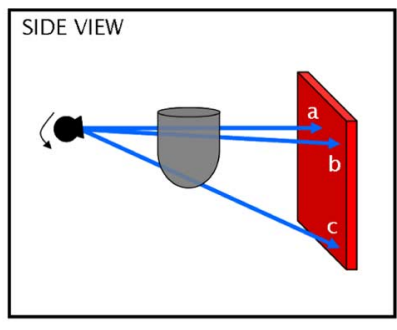

(b)
Fig. 2. The angles chosen in this study are consistent with a breast CT system with pendant geometry [9], [3] (a) described by Kwan et al. [6] with a $30 \times 40 \mathrm{~cm}$ detector, a source-to-isocenter distance of $44 \mathrm{~cm}$, and a source-to-detector distance of $88 \mathrm{~cm}$. (b) Location of selected X-ray landing locations to determine the angles.

on studying the response to a pinhole as a reasonable compromise between sufficient X-ray fluence collimated from a diagnostic X-ray tube beam, and little degradation of the point-like response that would indicate the asymmetries. For the comparison to be valid, we have included a model of the pinhole assembly into the geometry for the Monte Carlo simulations. The results, described in detail in a soon to appear paper, indicate that MANTIS is capable of predicting the magnitude of the spread in a pinhole response only by knowing geometry and properties of the materials and surfaces of the phosphor screen from scanning electron microscopy images. Preliminary results, expressed in terms of rms difference between simulated and experimental data, exhibit good agreement, much better than the comparison with single Gaussian fits to the experimental data. These results are promising, especially considering that the metric is very unforgiving since it captures the differences in the response over the entire image of the response on a pixel to pixel basis.

\section{RESULTS}

We selected three landing locations in the detector corresponding to angles of incidence of 1) normal incidence $\left.\left(\phi=0^{\circ}, \theta=0^{\circ}\right), 2\right)$ detector edge along the horizontal plane $\left(\phi=90^{\circ}, \theta=15^{\circ}\right)$, and 3) detector corner $\left(\phi=45^{\circ}, \theta=21.4^{\circ}\right)$, where $\phi$ is the polar angle and $\theta$ is the azimuthal angle for a coordinate system with the $x y$ plane aligned with the chest wall, and the $z$ axis pointing from the chest wall toward the breast nipple. A schematic of the geometry is presented in Fig. 2. These angles are meant to represent maximum angles that can be encountered in breast CT designs based on pendant geometry. Other designs with different geometries might result in different maximum angles of X-ray incidence.

In order to quantify the anisotropy generated by the oblique incidence of X-rays in breast CT systems, several methods were implemented. The first consisted of performing a 2-D asymmetric Gaussian fit on the detector response and calculating the corresponding elliptical aspect ratio. The fit was performed using the function GAUSS2DFIT from IDL (ITT Visual Information Solutions, Boulder, CO). The second approach was to fit an elliptical curve to the data points that define isocontours at different heights of the response $(0.01,0.1,0.25$, and 0.5$)$ and calculating the corresponding elliptical aspect ratio. In other 

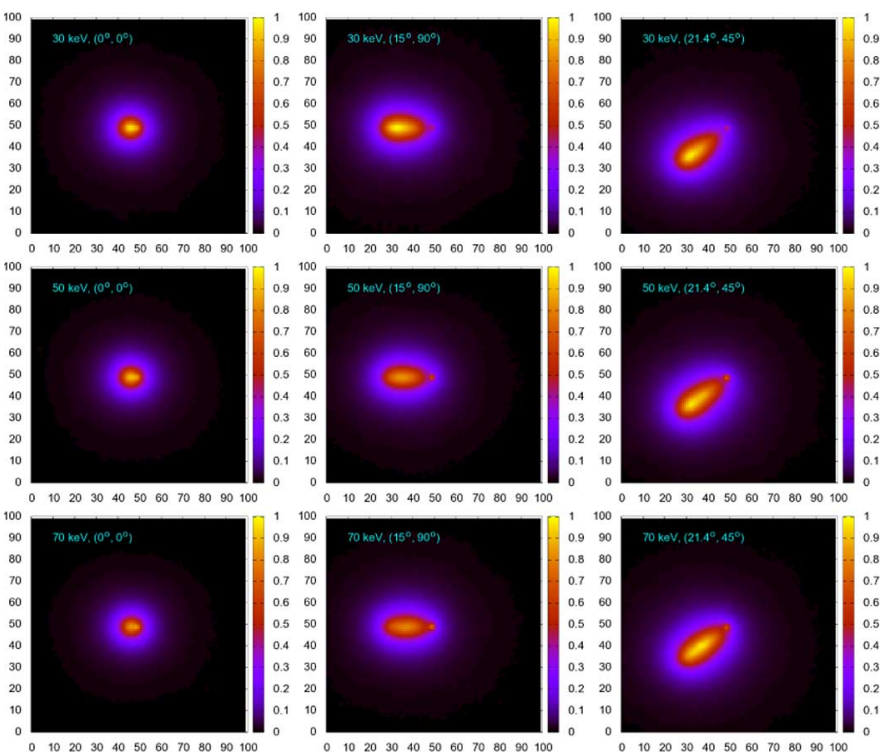

Fig. 3. Detector responses to monoenergetic X-rays at different locations in the detector (with different incidence angles). Image dimensions: $100 \times 10010-\mu \mathrm{m}$ pixels.

words, from the raw Monte Carlo data, one can extract data points that are located close (within some tolerance) to the desired height. This technique yields a set of points determined by a $(x, y)$ coordinate pair that are then used to locate the best elliptical function that describes the distribution of points in the $x y$ plane. The fit is performed using a numerically stable, noniterative algorithm for fitting ellipses to contour data points based on the method developed by Halir et al. [37]. The approach is based on noniterative least squares minimization and it is applicable to scattered or noisy data.

This second approach can be performed on isocontours generated from the Monte Carlo results, or on isocontours generated from the 2-D Gaussian fit. As we will show, the three approaches yield slightly different results but are in general concordance with respect to the overall trends seen in the data.

Fig. 3 shows the detector responses to monoenergetic X-rays at different incidence angles. The response is presented as a $100 \times 100$-pixel image with $10-\mu \mathrm{m}$ pixels obtained by binning the Monte Carlo results in appropriate spatial bins. The isocontours corresponding to the raw Monte Carlo results are shown in Fig. 4. To carry out the other approach of using fitted data, we performed the 2-D asymmetric Gaussian fits (presented as centered and rotated realizations of the Gaussian functions in Fig. 5), and the corresponding isocontour curves shown in Fig. 6. Note that even at normal incidence, the response functions do not generate a centered maximum. This effect might be attributed to the columnar tilt in the phosphor layer. Fig. 7 presents a summary of the aspect ratios of elliptical fits for a single 2-D Gaussian and for elliptical fits to the isocontour lines for different response heights. The maximum width of the detector response at all angles and energies considered with respect to the average width at normal incidence are plotted in Fig. 8. The additional elliptical curve fits to Monte Carlo raw
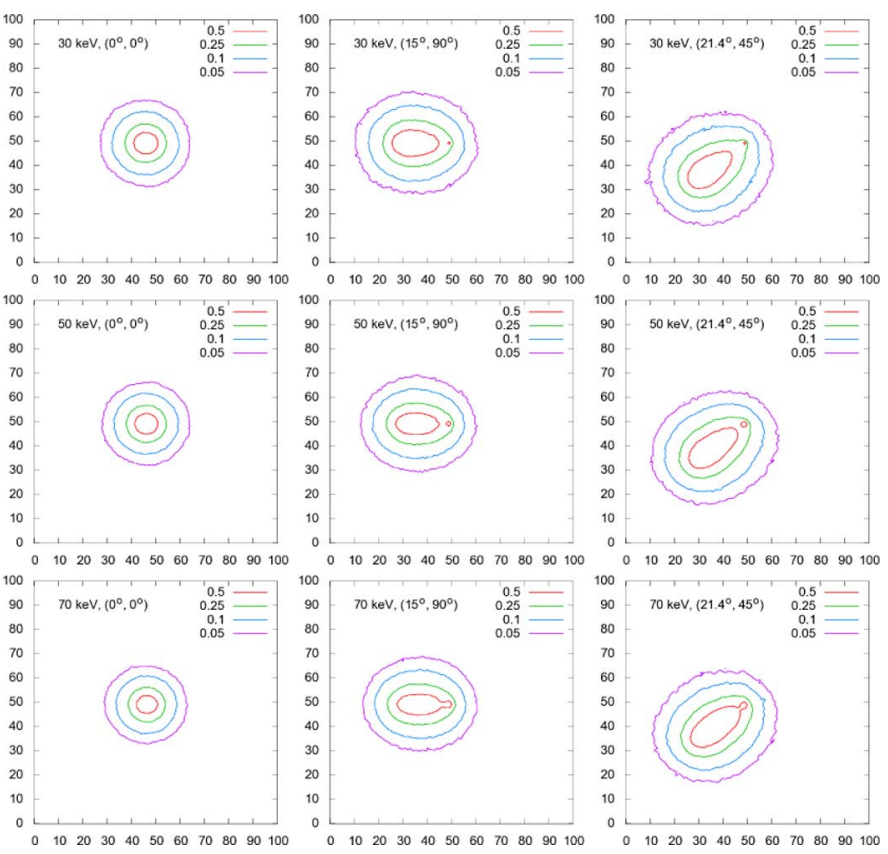

Fig. 4. Isocontour lines for the detector responses to monoenergetic X-rays at different locations in the detector (with different incidence angles). Image dimensions: $100 \times 10010-\mu \mathrm{m}$ pixels.
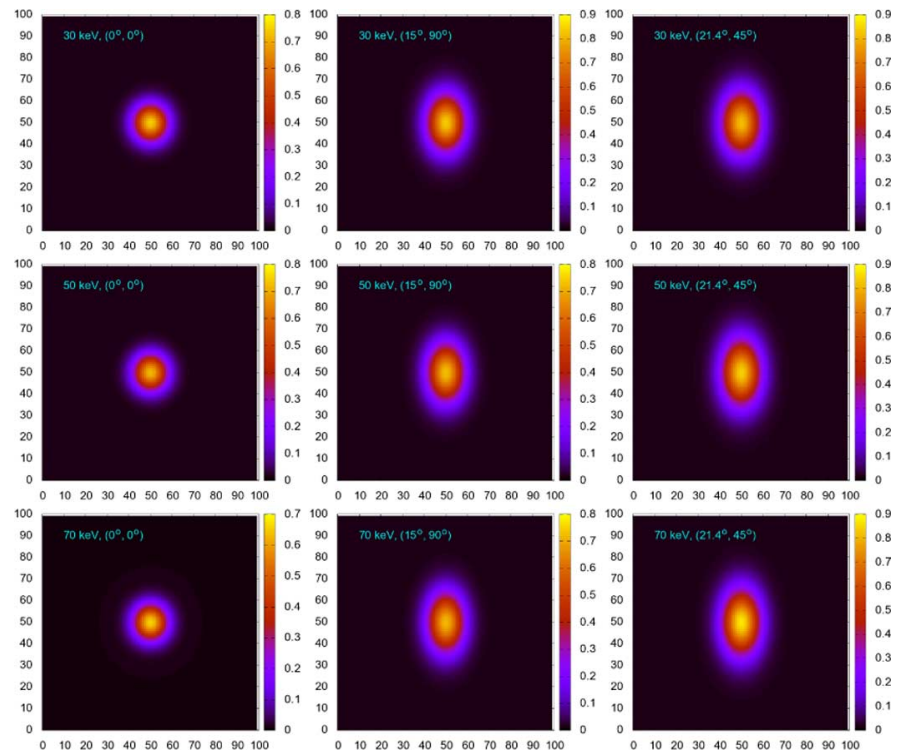

Fig. 5. Gaussian fits (centered and rotated) to the detector responses to monoenergetic X-rays at different locations in the detector (with different incidence angles). Image dimensions: $100 \times 10010-\mu \mathrm{m}$ pixels.

isocontour data, along with the results for the angular rotation, centering shifts, and axial dimensions, are shown in Fig. 9.

We summarize the results to highlight relevant figures with respect to the asymmetry of the phosphor response and with respect to the increase in blur due to oblique X-ray incidence in Tables II and III. Table II shows the maximum aspect ratio of the responses for the different calculation methods and across all energies and angles considered in this work. The maximum aspect ratios reach 2.53 when the calculation is performed with elliptical fits at the 0.5 isocontour level. The results suggest that 

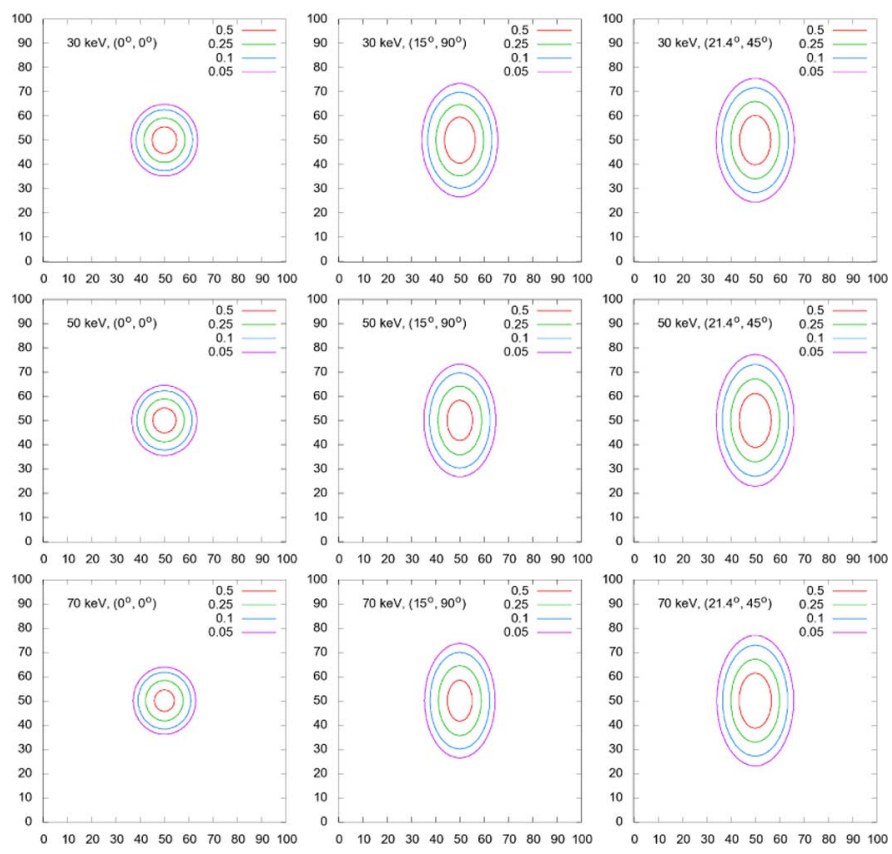

Fig. 6. Isocontour lines for the centered and aligned Gaussian fits to detector responses to monoenergetic X-rays at different locations in the detector (with different incidence angles). Image dimensions: $100 \times 10010-\mu \mathrm{m}$ pixels.

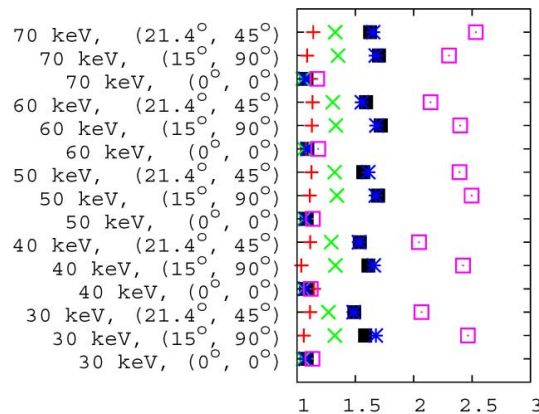

Aspect ratio of elliptical fits

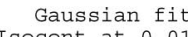

Isocont at 0.25

Isocont at 0.50

Fig. 7. Aspect ratio of the elliptical fits for a single 2-D Gaussian and for elliptical fits to the isocontour lines at different heights of the response $(0.01,0.1$, 0.25 , and 0.5 ).

the asymmetry becomes more important at higher levels of the response, towards the peak. Table III shows the maximum increase in phosphor blur with respect to normal incidence for the energies studied in this work. A maximum increase of 1.86 is seen at $50 \mathrm{keV}$ with no definitive trend with respect of X-ray energy.

\section{DISCUSSION}

Our results suggest that the asymmetry in the response of phosphor screens for X-ray digital detectors does not change significantly with X-ray energy. When energy changes (within the range considered in this work), the location of the maximum of each response shifts slightly. This shift is a very high spatial frequency effect likely to be hidden by large area, discrete pixel integration. Some of the detector responses we present in this paper have dual peak structure (within a $50 \mu \mathrm{m}$ distance) due to the complex particle and optical transport that occurs in the

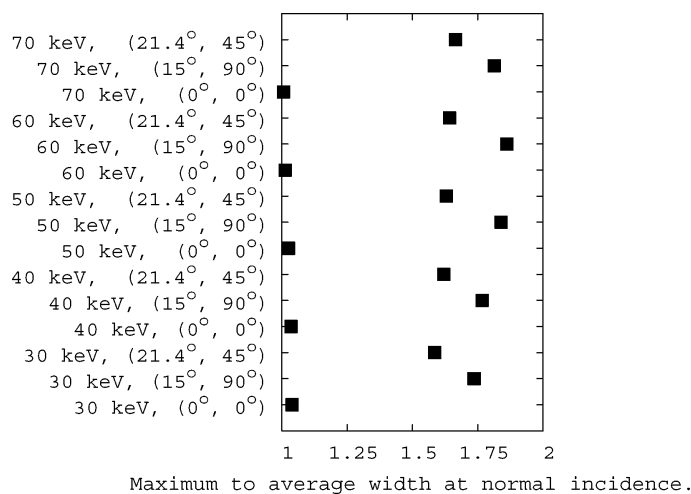

Fig. 8. Ratio between the maximum width of the detector response with respect to the average width of the response at normal incidence.

columnar structure in $\mathrm{CsI}(\mathrm{Tl})$ detectors. This effect is also likely to be insignificant given the large pixel sizes used in CT detectors.

As seen in Table II, the maximum aspect ratio calculated by any of our methods is 2.53 which constitutes a significant departure from the assumption of isotropy in the phosphor blur. In addition, increases in blur seen with respect to normal X-ray incidence up to 1.86 suggest that the assumption of shift-invariance might not be warranted in this type of detectors. This is further substantiated by the results shown in Fig. 9 where the ratio of the elliptical axes $\left(r_{a} / r_{b}\right)$ reaches 0.39 corresponding to an increase in blur of 2.56 .

In addition to the increase in detector blur, we present results with respect to the spatial shift of the response measured as $x$ and $y$ displacements of the maxima. The results, shown in Fig. 10, demonstrate that, as postulated by Mainprize and collaborators [8], the shift of the response due to oblique X-ray incidence is significant (in the order of $100 \mu \mathrm{m}$ ). Knowledge about these shifts for specific breast CT designs will contribute to improved image quality by properly accounting for it in the image postprocessing and reconstruction algorithm.

Our findings in terms of widths, shifts, and asymmetries of the detector response have to be analyzed considering the overall system performance, including pixel discretization and detector electronics and readout, focal spot and tube motion blur, and eventual reconstruction algorithm for presentation to the human reader. For this, it is important to note that the results obtained for the detector response do not consider the pixel discretization which is of the order of a few hundred microns for typical breast CT systems being investigated today. Nevertheless, the resolution property of the detector before pixelation is an important design parameter. In fact, the ideal pixel dimensions for a breast CT system have not yet been fully investigated. Our findings for the "presampled" spread and shift of the oblique X-ray response might contribute to the development of optimization approaches that include discrete pixel effects.

The results presented in this paper need experimental verification that depends on the specific structures of the detector being studied. However, the experimental verification of these results is not straightforward due to the complications of performing the measurements. For instance, edge methods to measure resolution are limited to a uni- or bi-dimensional measurement of blur. Without a priori knowledge of the exact orientation of the response with respect to the photodiode array, the presampled modulation transfer function might not be aligned with the direction of the elliptical axes. An approach that relies on a circular object [38] using a high resolution optical array 

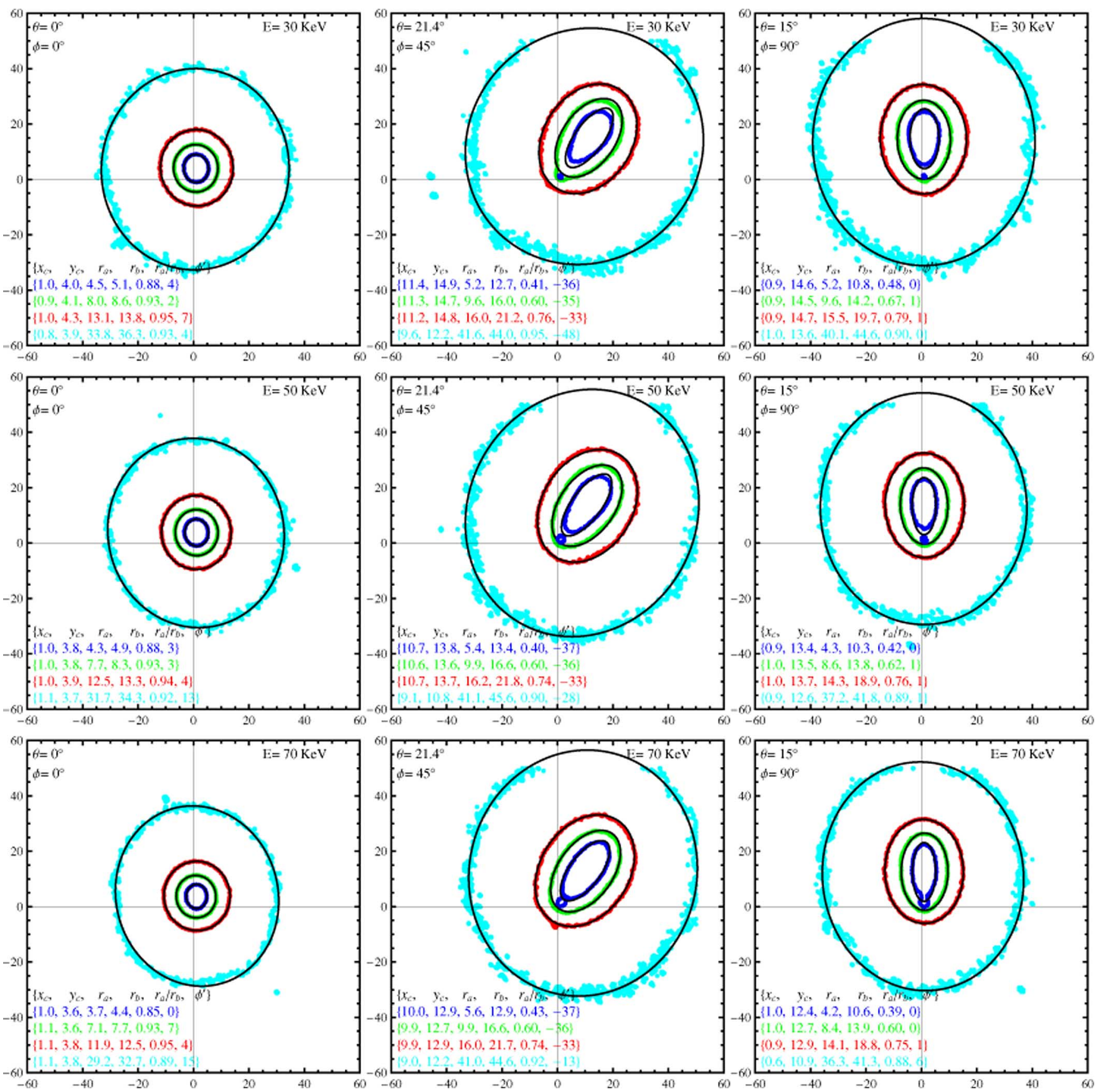

Fig. 9. Elliptical fits to the isocontours at different heights of the responses.

TABLE II

MaXimum Aspect Ratios For EACh CALCulation Method ACROSS ENERGIES AND ANGLES OF INCIDENCE

\begin{tabular}{lc}
\hline Method & Maximum aspect ratio \\
\hline 2D elliptical Gaussian & 1.72 \\
Elliptical fit at 0.01 & 1.14 \\
Elliptical fit at 0.10 & 1.35 \\
Elliptical fit at 0.25 & 1.68 \\
Elliptical fit at 0.50 & 2.53 \\
\hline
\end{tabular}

might provide the necessary level of detail to verify the result of the MANTIS simulations.

Knowledge of the response of the detector can be incorporated into the reconstruction methods, or used to apply adaptive
TABLE III

MAXIMUM INCREASE IN BLUR WITH RESPECT TO THE BLUR AT NORMAL INCIDENCE AT THAT ENERGY FOR DIFFERENT X-RAY ENERGIES

\begin{tabular}{lc}
\hline Energy $(\mathrm{keV})$ & Maximum increase in blur \\
\hline 30 & 1.74 \\
40 & 1.77 \\
50 & 1.84 \\
60 & 1.86 \\
70 & 1.81
\end{tabular}

algorithms to interpret the projection images. In this context, our paper is intended to provide a general understanding of the effect of oblique X-ray incidence in breast CT systems. The results of 


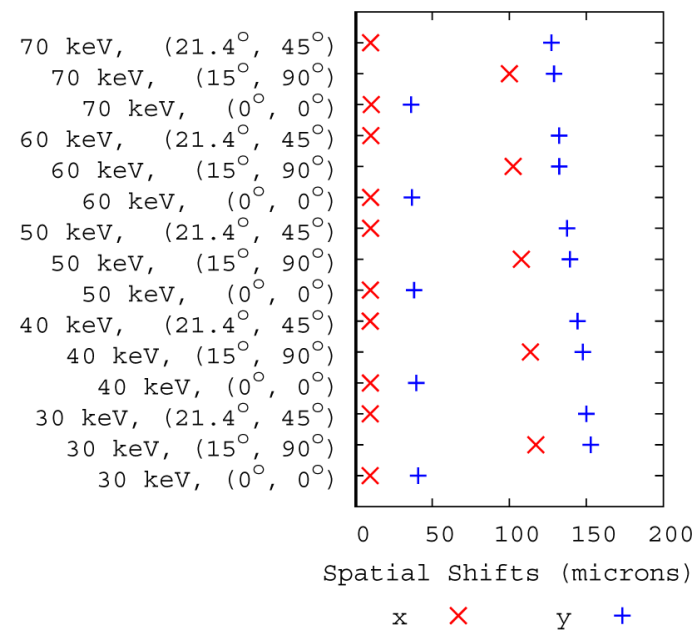

Fig. 10. Spatial shifts of the maximum in the detector response due to oblique $\mathrm{X}$-ray incidence.

this paper might guide developers of breast CT systems to consider a task-based assessment methodology for system design and optimization that does not rely on assumptions of shift-invariance.

\section{REFERENCES}

[1] X. Gong, S. J. Glick, B. Liu, A. A. Vedula, and S. Thacker, "A computer simulation study comparing lesion detection accuracy with digital mammography, breast tomosynthesis, and cone-beam CT breast imaging," Med. Phys. vol. 33, no. 4, pp. 1041-1052, 2006.

[2] J. M. Boone, N. Shah, and T. R. Nelson, "A comprehensive analysis of dgn[sub ct] coefficients for pendant-geometry cone-beam breast computed tomography," Med. Phys. vol. 31, no. 2, pp. 226-235, 2004.

[3] J. M. Boone, A. L. C. Kwan, J. A. Seibert, N. Shah, K. K. Lindfors, and T. R. Nelson, "Technique factors and their relationship to radiation dose in pendant geometry breast CT," Med. Phys. vol. 32, no. 12, pp. 3767-3776, 2005

[4] K. Yang, A. L. C. Kwan, and J. M. Boone, "Computer modeling of the spatial resolution properties of a dedicated breast CT system," Med. Phys. vol. 34, no. 6, pp. 2059-2069, 2007.

[5] A. L. C. Kwan, J. M. Boone, and N. Shah, "Evaluation of X-ray scatter properties in a dedicated cone-beam breast CT scanner," Med. Phys. vol. 32, no. 9, pp. 2967-2975, 2005.

[6] A. L. C. Kwan, J. M. Boone, K. Yang, and S.-Y. Huang, "Evaluation of the spatial resolution characteristics of a cone-beam breast CT scanner," Med. Phys. vol. 34, no. 1, pp. 275-281, 2007.

[7] A. Badano, I. S. Kyprianou, R. J. Jennings, and J. Sempau, "Anisotropic imaging performance in breast tomosynthesis," Med. Phys., vol. 34, no. 11, pp. 4076-4091, 2007.

[8] J. G. Mainprize, A. K. Bloomquist, M. P. Kempston, and M. J. Yaffe, "Resolution at oblique incidence angles of a flat panel imager for breast tomosynthesis," Med. Phys. vol. 33, no. 9, pp. 3159-3164, 2006.

[9] S. J. Glick, S. Thacker, X. Gong, and B. Liu, "Evaluating the impact of X-ray spectral shape on image quality in flat-panel CT breast imaging," Med. Phys. vol. 34, no. 1, pp. 5-24, 2007.

[10] F. Salvat, J. M. Fernández-Varea, and J. Sempau, PENELOPE, A code system for Monte Carlo simulation of electron and photon transport. Issy-les-Moulineaux, France: OECD Nuclear Energy Agency, 2003.

[11] J. Sempau, E. Acosta, J. Baró, J. M. Fernández-Varea, and F. Salvat, "An algorithm for Monte Carlo simulation of coupled electron-photon transport," Nucl. Instrum. Methods Phys. Res. Section B: Beam Interactions Materials Atoms, vol. 132, no. 3, pp. 377-390, 1997.

[12] A. Badano and J. Sempau, "Combined X-ray, electron, and optical Monte Carlo simulations of indirect radiation imaging systems," Phys. Med. Biol., vol. 51, pp. 1545-1561, 2006.

[13] A. Badano, I. S. Kyprianou, and J. Sempau, "Anisotropic imaging performance in indirect X-ray imaging detectors," Med. Phys. vol. 33, no. 8, pp. 2698-2713, 2006.

[14] E. A. Siegbahn, B. Nilsson, J. M. Fernández-Varea, and P. Andreo, "Calculations of electron fluence correction factors using the Monte Carlo code PENELOPE," Phys. Med. Biol., vol. 48, pp. 1263-1275, 2003.
[15] C. DesRosiers, V. Moskvin, A. F. Bielajew, and L. Papiez, "150-250 $\mathrm{MeV}$ electron beams in radiation therapy," Phys. Med. Biol., vol. 45, pp. 1781-1805, 2000.

[16] J. Mazurier, J. Gouriou, B. Chauvenet, and J. Barthe, "Calculation of perturbation correction factors for some reference dosimeters in highenergy photon beams with the Monte Carlo code PENELOPE," Phys. Med. Biol., vol. 46, pp. 1707-1717, 2001.

[17] F. Mourtada, C. G. Soares, S. M. Seltzer, P. M. B. Jr., J. M. Fernández-Varea, J. Asenjo, and S. H. Lott, "Dosimetry characterization of a ${ }^{32} \mathrm{P}$ source wire used for intravascular brachytherapy with automated stepping," Med. Phys. vol. 30, no. 5, pp. 959-971, 2003.

[18] F. M. Al-Dweri and A. M. Lallena, "A simplified model of the source channel of the Leksell Gamma Knife: Testing multisource configurations with PENELOPE," Phys. Med. Biol., vol. 49, no. 15, pp. 3441-53, 2004.

[19] A. Cot, J. Sempau, D. Pareto, S. Bullich, J. Pavia, F. Calviño, and D. Ros, "Study of the point spread function (PSF) for ${ }^{123}$ I SPECT imaging using Monte Carlo simulation," Phys. Med. Biol., vol. 49, no. 14, pp. 3125-36, 2004.

[20] V. Moskvin, R. Timmerman, C. DesRosiers, M. Randall, P. DesRosiers, P. Dittmer, and L. Papiez, "Monte Carlo simulation of the Leksell Gamma Knife: II. Effects of heterogeneous versus homogeneous media for stereotactic radiosurgery," Phys. Med. Biol., vol. 49, no. 21, pp. 4879-95, 2004.

[21] J. Sempau, P. Andreo, J. Aldana, J. Mazurier, and F. Salvat, "Electron beam quality correction factors for plane-parallel ionization chambers: Monte Carlo calculations using the PENELOPE system," Phys. Med. Biol., vol. 49, no. 18, pp. 4427-44, 2004.

[22] J. Sempau, J. M. Fernández-Varea, E. Acosta, and F. Salvat, "Experimental benchmarks of the Monte Carlo code PENELOPE," Nucl. Instrum. Methods Phys. Res. Section B: Beam Interactions Materials Atoms, vol. 207, no. 2, pp. 107-123, 2003.

[23] P. Carrasco, N. Jornet, M. A. Duch, L. Weber, M. Ginjaume, T. Eudaldo, D. Jurado, A. Ruiz, and M. Ribas, "Comparison of dose calculation algorithms in phantoms with lung equivalent heterogeneities under conditions of lateral electronic disequilibrium," Med. Phys. vol. 31, no. 10, pp. 2899-2911, 2004.

[24] X. Llovet, L. Sorbier, C. S. Campos, E. Acosta, and F. Salvat, "Monte Carlo simulation of X-ray spectra generated by kilo-electron-volt electrons," J. Appl. Phys. vol. 93, no. 7, pp. 3844-3851, 2003.

[25] A. Badano, "Image quality degradation by light scattering processes in high performance display devices for medical imaging," Ph.D. dissertation, Univ. Michigan, Ann Arbor, 1999.

[26] A. Badano, M. J. Flynn, E. Muka, K. Compton, and T. Monsees, "The veiling glare point-spread function of medical imaging monitors," Proc. SPIE, vol. 3658, pp. 458-467, 1999.

[27] A. Badano and M. J. Flynn, "Monte Carlo modeling of the luminance spread function in flat panel displays," in Proc. Int. Display Res. Conf., 1997, vol. 21, pp. 382-385.

[28] A. Badano and J. Kanicki, "Monte Carlo modeling method for light transport in organic thin-film light-emitting devices," in Proc. OSA Conf. Organic Thin Films Photonics Appl., 1999, pp. 205-207.

[29] A. Badano and J. Kanicki, "Monte Carlo analysis of the spectral photon emission and extraction efficiency of organic light-emitting devices," $J$. Appl. Phys., vol. 90, no. 4, pp. 1827-1830, 2001.

[30] G. F. Knoll and T. F. Knoll, "Light collection in scintillation detector composites for neutron detection," IEEE Trans. Nucl. Sci., vol. 35, no. 1, pp. 872-875, Feb. 1988.

[31] A. Badano, "Optical blur and collection efficiency in columnar phosphors for X-ray imaging," Nucl. Instrum. Methods Phys. Res. Part A, vol. 508, no. 3, pp. 467-479, 2003.

[32] J.-M. Levy-Leblond and F. Balibar, Quantics. : : North-Holland, 1990.

[33] J. A. Rowlands and J. Yorskton, Flat Panel Detectors for Digital Radiography. Bellingham, WA: SPIE Press, 2000, pp. 223-328.

[34] A. Badano, I. Kyprianou, K. H. Tang, and A. Saha, "Validation of simulated point-response functions in columnar phosphor screens," Proc. SPIE, vol. 6142, p. 65100Y1-5, 2006.

[35] M. Freed, S. Miller, K. Tang, and A. Badano, "Experimental validation of Monte Carlo (MANTIS) simulated x-ray response of columnar CsI phosphor screen," Med. Phys., 2009, submitted for publication.

[36] I. S. Kyprianou, A. Badano, S. Park, B. Gallas, L. Thompson, and K. J. Myers, "A practical method for measuring the $\mathrm{H}$ matrix of digital X-ray and cone beam CT imaging systems," Proc. SPIE, vol. 6142, 2006.

[37] R. Halir and J. Flusser, "Numerically stable direct least squares fitting of ellipses," in Proc. Int. Conf. Central Eur. Comput. Graphics, Visualizat. Interactive Digital Media, 1998, pp. 125-132.

[38] K. A. Fetterly, N. J. Hangiandreou, B. A. Schueler, and E. R. Ritenour, "Measurement of the presampled two-dimensional modulation transfer function of digital imaging systems," Med. Phys. vol. 29, no. 5, pp. 913-921, 2002. 